\title{
DIFFERENCES OF FUNCTIONS AND MEASURES
}

\author{
R. E. EDWARDS
}

(Received 26 September 1966, revised 2 March 1967)

\section{Introduction and statement of results}

Throughout this paper, $G$ will denote a locally compact Hausdorff group with a chosen left Haar measure $m$. For each $s \in G, \tau_{s}$ denotes the left translation operator which acts on functions $f$ according to the rule $\tau_{s} f(x)=f\left(s^{-1} x\right)$ and on measures or distributions in the corresponding way. The associated left difference operator $\tau_{s}-1$ is denoted by $\Delta_{s}$. On occasions it will be more convenient to write $\tau(s)$ and $\Delta(s)$ in place of $\tau_{s}$ and $\Delta_{s}$.

The type of problem to be considered is as follows: Suppose that $f$ is a function, measure or distribution on $G$ with the property that, for each $s$ belonging to a not-too-thin subset $S$ of $G$, the difference $\Delta_{s} f$ (or an iterated difference $\Delta_{s}^{n} f$ ) belongs to some pre-assigned function space. What can then be said about $f$ ? In particular, when can one infer that $f$ itself belongs to the same function space?

N. G. de Bruijn [1], [2] obtained many results of this type, both positive and negative in nature, for the case in which $G=S=R$ (the additive group of reals). De Bruijn's results have been significantly extended in certain directions by Kemperman [14] and Carroll [15], [16] (for reference to which the present writer is indebted to a referee). The results in Edwards [3] are of a somewhat similar nature, the hypotheses there being that $m_{*}(S)$ (the supremum of $m(K)$ for compact sets $K C S$ ) is positive and that the set of differences $\left\{\Delta_{s} f: s \in S\right\}$ is relatively compact in a suitable sense.

The aims and methods of de Bruijn, Kemperman and Carroll are, however, somewhat different from those of the present paper. They are concerned to some extent with the pathology of non-measurable functions and with that of noncomplete function spaces. With these aspects we are never concerned; we shall assume outright that the functions are at least locally integrable (although we do admit non-functions in the shape of measures or distributions), and we make full use of the fact that the function spaces considered are complete.

An example illustrates well the difference in viewpoint. De Bruijn ([1], pp. 211-212) shows that if $f \in R^{R}$ is such that $\Delta_{s} f \in L_{\mathrm{loc}}^{2}(R)$ for each $s \in R$, 
then $f=g+h+k$, where $g \in L_{\text {loc }}^{2}(R), h$ is additive and $k$ is such that $\Delta_{s} k=0$ a.e. for each $s \in R$. The analogous problem with $L_{\text {loc }}^{p}$ in place of $L_{\text {loc }}^{2}$ is left open. We, on the other hand, will show (as a corollary of Theorems 3 and 5) that if $1 \leqq p \leqq \infty$, and if $f$ is a distribution on $R$ with the property that $\Delta_{s} f \in L_{\mathrm{loc}}^{p}(R)$ for each $s$ belonging to a set $S \subset R$ which is either nonnull or contains a nonmeagre $F_{\sigma}$-set, then $f \in L_{\mathrm{loc}}^{p}(R)$. Although it is true that special cases of this corollary of Theorems 3 and 5 are derivable from de Bruijn's result with little trouble, this approach is limited in scope and (we feel) not the natural one.

Our positive results, as well as coping with general values of the exponent $p$ in the range $[1, \infty]$, also apply to cases in which the data involves iterated differences $\Delta_{s}^{n} f$ of arbitrarily high orders.

A few negative results are obtained, applying in cases where the set $S$ is locally negligible or meagre but may well be perfect and uncountable. Here again the examples are quite different in nature from those of de Bruijn insofar as we countenance no dealings with nonmeasurable functions.

Immediately following a description of notations are the statements of the positive results, the proofs of which occupy $\S \S 2$ and 4 . The negative results are considered in $\S 5$.

Notation. The term "measure" will always mean "Radon measure" and $M(G)$ will denote the space of measures on $G$. If $\Omega$ is a nonvoid open subset of $G, C_{c}(\Omega)$ denotes the space of continuous functions on $G$ with compact supports contained in $\Omega$.

For simplicity we shall, when speaking of distributions, assume that the underlying group $G$ is a Lie group. In such cases $\mathscr{D}^{\prime}(G)$ denotes the space of distributions on $G$; and, for any nonvoid open subset $\Omega$ of $G, C_{\mathbf{c}}^{\infty}(\Omega)$ denotes the space of indefinitely differentiable functions on $G$ with compact supports contained in $\Omega$. The space of indefinitely differentiable functions on $G$ is denoted by $C^{\infty}(G)$.

The usual Lebesgue spaces $L^{p}(G)$ and $L_{\mathrm{loc}}^{p}(G)$ are constructed relative to left Haar measure $m$ on $G$, the exponent $p$ satisfying always $1 \leqq p \leqq \infty$. These, and the other function spaces already mentioned, may and will be injected into $M(G)$ in a familiar way; when $G$ is a Lie group we go one step further by injecting $M(G)$ itself into $\mathscr{D}^{\prime}(G)$.

If $E$ is a subset of $G$ and $r$ a positive integer, $E^{1 / r}$ denotes the set of $x \in G$ satisfying $x^{r} \in E$. At times we shall need to impose upon $G$ one or more of the following conditions:

$\left(C_{r}\right)$ There exists a number $c_{r}>0$ such that

$$
m\left(E^{1 / r}\right) \leqq c_{r} \cdot m(E)
$$

for each relatively compact measurable subset $E$ of $G$. 
Evidently, the condition $\left(C_{1}\right)$ is void.

If $r$ is an integer exceeding 1 , and if $j_{r}$ denotes the map $x \rightarrow x^{r}$ of $G$ into itself, then $j_{r}$ is a continuous homomorphism of $G$ and $E^{1 / r}=j_{r}^{-1}(E)$. If $G$ is divisible ( $[13]$, p. 440), then $j_{r}(G)=G$ for all $r$; and $G$ is torsion-free ([13], p. 439) if and only if $j_{r}$ is $1-1$ for all $r$. Hence, if $G$ is $\sigma$-compact, locally countably compact, divisible, and torsion-free, then ([13], (5.29), p. 42) $j_{r}^{-1}$ is continuous. In any case, continuity of $j_{\tau}^{-1}$ will suffice to ensure that $\left(C_{r}\right)$ is satisfied (thanks to the essential uniqueness of left Haar measure).

STATEMENTS OF THE POSITIVE RESULTS. Some of these apply to measures only, others to distributions.

Theorem 1. Suppose that $1<p \leqq \infty$, that $\mu \in M(G)$, and that

$$
S=\left\{s \in G: \Delta_{s} \mu \in L_{\mathrm{loc}}^{p}(G)\right\}
$$

satisfies either (i) $m_{*}(S)>0$ or (ii) $S$ is nonmeagre. Then $\mu \in L_{\mathrm{loc}}^{p}(G)$.

THEOREM 2. Suppose that $p$ and $\mu$ are as in Theorem 1. Suppose further that $G$ satisfies $\left(C_{r}\right)$ for each positive integer $r$, and that

$$
S=\left\{s \in G: \Delta_{s}^{n} \mu \in L_{\mathrm{loc}}^{p}(G) \text { for some positive s-dependent integer } n\right\}
$$

satisfies either (i) or (ii) of Theorem 1. Then again one may infer that $\mu \in L_{\mathrm{loc}}^{p}(G)$.

Theorem 3. Suppose that $G$ is a Lie group. A true statement results if, in Theorem 1, the hypothesis $\mu \in M(G)$ is replaced by $\mu \in \mathscr{D}^{\prime}(G)$.

THEOREM 4. Suppose that $G$ is a Lie group satisfying $\left(C_{r}\right)$ for every positive integer $r$, that $1<p \leqq \infty$, that $\mu \in \mathscr{D}^{\prime}(G)$, and that $S$ is defined as in Theorem 2. If $S$ is nonmeagre, then $\mu \in L_{\mathrm{loc}}^{p}(G)$. we have

Concerning the case $p=1$, excluded from the preceding theorems,

THEOREM 5. Suppose that $p=1$. The conclusions of Theorems 1 and 2 again follow on the basis of hypothesis (i), and the conclusion of Theorem 1 follows on the basis of the hypothesis (ii') $S$ contains a nonmeagre $F_{\sigma}$-set.

If $G$ is a Lie group, these extensions are valid if the hypothesis $\mu \in M(G)$ be replaced by $\mu \in \mathscr{D}^{\prime}(G)$.

In connection with Theorem 5 we observe that (in any locally compact Hausdorff space $G$ ) any nonvoid open set $U$ is nonmeagre (see [4], 0.2.18 and 0.3.16); and that any such set $U$ contains a nonvoid open (and therefore nonmeagre) $F_{\sigma}$-set $V$. On the other hand, a nonmeagre set $S$ may contain no nonmeagre $F_{\sigma}$-set (as when $G=R$ and $S$ is the set of irrationals).

The techniques used to prove Theorems $1-5$ can be adapted so as to 
apply to cases in which $L_{\text {loc }}^{p}(G)$ is replaced by certain other spaces, among which are:

(a) $L^{p}(G)$ - provided we assume in advance that $\mu$ is a bounded measure or, if a distribution, belongs to $\mathscr{D}_{L^{p}(G)}^{\prime}$ (see [5], Tome II, p. 56);

(b) $C^{l}(G)$ with $l$ a nonnegative integer or $\infty, G$ being a Lie group.

\section{Preliminaries}

2.1 If $g \in L_{c}^{1}(G)$, the set of integrable functions on $G$ having compact supports, $\mu \in M(G)$ and $r$ is a nonnegative integer, we define $g_{*}^{(r)} \mu \in M(G)$ by the vector-valued integral

$$
g_{*}^{(r)} \mu=\int_{G} g(s) \cdot \tau\left(s^{r}\right) \mu \cdot d s ;
$$

here and elsewhere we shall write $\int_{G} \cdots d s$ in place of $\int_{G} \cdots d m(s)$ for integrals with respect to left Haar measure $m$. The existence of the integral in (2.1.1) is assured by 8.16.1 and 8.16 .3 of [4]. In particular,

$$
\begin{aligned}
& g_{*}^{(0)} \mu=\int_{G} g(s) d s \cdot \mu, \\
& g_{*}^{(1)} \mu=g * \mu,
\end{aligned}
$$

where $g * \mu$ is the usual convolution of $g$ and $\mu$ (cf. [4], p. 568). In view of the formula

it appears that

$$
\Delta_{s}^{n} \mu=\sum_{r=0}^{n}(-1)^{n-r}{ }_{n} C_{r} \tau\left(s^{r}\right) \mu,
$$

$$
\int_{G} g(s) \cdot \Delta_{s}^{n} \mu \cdot d s=\sum_{r=0}^{n}(-1)^{n-r}{ }_{n} C_{r} g_{*}^{(r)} \mu
$$

for $g \in L_{c}^{1}(G)$ and $\mu \in M(G), n$ denoting any positive integer.

\subsection{Properties of $g_{*}^{(r)} \mu$.}

(a) It is well known that $g_{*}^{(1)} \mu=g * \mu \in L_{\text {loc }}^{p}(G)$ whenever $g \in L_{c}^{p}(G)$, $\mu \in M(G)$, and $1 \leqq p \leqq \infty$.

(b) If $G=R^{m}$, it is easy to see that

$$
g_{*}^{(r)} \mu=g_{r} * \mu
$$$$
(r=1,2, \cdots),
$$

where $g_{r}(x)=r^{-1} g\left(r^{-1} x\right)$. This in turn makes it clear that $g_{*}^{(r)} \mu \in L_{\mathrm{loc}}^{p}\left(R^{m}\right)$ whenever $g \in L_{c}^{p}\left(R^{m}\right), \mu \in M\left(R^{m}\right), r=1,2, \cdots$ and $1 \leqq p \leqq \infty$. We need to know that the last statement is true for any $G$ satisfying condition $\left(C_{r}\right)$ for every $r$.

In any case, $\left(C_{r}\right)$ ensures that

$$
\left\|\psi^{r}\right\|_{p} \leqq c_{r}^{\prime} \cdot\|\psi\|_{p}
$$


for any $\psi \in C_{c}(G)$, where $\psi^{r}$ is defined by

$$
\psi^{r}(x)=\psi\left(x^{r}\right)
$$

and where $r$ is a positive integer.

On the other hand the action of the measure $g_{*}^{(r)} \mu$ on the function $\phi \in C_{c}(G)$ is specified by

$$
\begin{aligned}
\left\langle\phi, g_{*}^{(r)} \mu\right\rangle & =\int_{G} g(s) \cdot\left\langle\phi, \tau\left(s^{r}\right) \mu\right\rangle d s \\
& =\int_{G} g(s) d s \int_{G} \phi\left(s^{\tau} x\right) d \mu(x) \\
& =\int_{G} d \mu(x) \int_{G} \phi\left(s^{r} x\right) g(s) d s .
\end{aligned}
$$

Now (2.2.2) and Hölder's inequality shows that the inner integral in (2.2.3) tends to zero in $C_{c}(G)$ as $\phi \rightarrow 0$ in $L_{c}^{p^{\prime}}(G)$, where (as usual) $p^{\prime}$ is the conjugate exponent defined by $1 / p+1 / p^{\prime}=1$. If $p>1$, this shows that indeed $g_{*}^{(\tau)} \mu \in L_{\mathrm{loc}}^{p}(G)$, and this case is thus disposed of.

Consider next the case in which $p=1$. Here it suffices (see [6], $\S 5$ ) to show that to each relatively compact open set $\Omega \subset G$ and each number $\varepsilon>0$ corresponds a number $c=c(\Omega, \varepsilon)$ such that

$$
\left|\left\langle\phi, g_{*}^{(r)} \mu\right\rangle\right| \leqq \varepsilon \cdot\|\phi\|_{\infty}+c \cdot\|\phi\|_{1}
$$

for functions $\phi \in C_{c}(G)$ with support contained in $\Omega$. In view of (2.2.3) and the fact that the inner integral appearing therein vanishes outside a compact set $K$ depending only on $\Omega$ and the support of $g$, to prove (2.2.4) it is enough to show that for $x \in K$ one has

$$
I \equiv\left|\int_{G} \phi\left(s^{r} x\right) g(s) d s\right| \leqq \varepsilon \cdot\|\phi\|_{\infty}+c^{\prime} \cdot\|\phi\|_{1}
$$

under the same hypotheses on $\phi$. However, a number $k$ can be chosen so large that, if $E_{k}$ is the set of $s \in G$ at which $|g(s)|>k$, then

$$
\int_{E_{k}}|g(s) d s| \leqq \varepsilon
$$

Consequently one has for $x \in K$

$$
\begin{aligned}
I & \leqq\left\{\int_{E_{k}}+\int_{G \mid E_{k}}|| \phi\left(s^{r} x\right)|\cdot| g(s) \mid d s\right. \\
& \leqq \varepsilon \cdot\|\phi\|_{\infty}+k \int_{G}\left|\phi\left(s^{r} x\right)\right| d s \\
& \leqq \varepsilon \cdot\|\phi\|_{\infty}+k^{\prime} \cdot\left\|\phi^{r}\right\|_{1},
\end{aligned}
$$

where $k^{\prime}$ absorbs a factor depending on the supremum on $K$ of the modular function of $G$. Using condition $\left(C_{r}\right),(2.2 .5)$ is now obtainable with $c^{\prime}=k^{\prime} c_{1}$.

(c) If $G$ is a Lie group and $\mu$ is any distribution on $G$, the formula 
(2.1.1) defines $g_{*}^{(r)} \mu$ as an element of $\mathscr{D}^{\prime}(G)$. In this case it is very simple to see that $g_{*}^{(r)} \mu \in C^{\infty}(G)$ whenever $g \in C_{c}^{\infty}(G)$ and $r$ is a positive integer.

2.3 The functions $N_{p, \Omega}$. If $1<p \leqq \infty$ and $\Omega$ is a relatively compact open subset of $G, N_{p, Q}$ is defined as a function from $M(G)$ into $[0, \infty]$ by the formula

$$
N_{p, \Omega}(\mu)=\operatorname{Sup}|\langle\phi, \mu\rangle|,
$$

where the supremum is taken relative to those functions $\phi \in C_{c}(\Omega)$ satisfying $\|\phi\|_{p^{\prime}} \leqq 1$. If $G$ is a Lie group, and $\mu \in \mathscr{D}^{\prime}(G)$, we modify the definition of $N_{p, \mu}(\Omega)$ by demanding that $\phi \in C_{c}^{\infty}(\Omega)$.

It can be seen without difficulty (cf. [3], p. 404) that, if $1<p \leqq \infty$, the restriction $\mu \mid \Omega$ belongs to $L^{p}(\Omega)$ if and only if $N_{p, \Omega}(\mu)<\infty$, in which case $N_{p, \Omega}(\mu)$ is equal to the $L^{p}(\Omega)$-norm of $\mu \mid \Omega$. Hence, $\mu \in L_{\text {loc }}^{p}(G)$ if and only if $N_{p, \Omega}(\mu)<\infty$ for each relatively compact open subset $\Omega$ of $G$.

The preceding assertions are no longer valid when $p=1$, which is why this case demands separate treatment at various points. It remains true, however, to assert $N_{1, \Omega}(\mu)$ coincides with the $L^{1}(\Omega)$-norm of $\mu \mid \Omega$ whenever $\mu \in L_{\mathrm{loc}}^{\mathbf{1}}(G)$.

2.4 In the course of the proofs of Theorems $1-4$ we shall be confronted with the situation in which $\mu \in M(G)$ or $\mathscr{D}^{\prime}(G), 1<p \leqq \infty$, and

$$
N_{p, \Omega}\left(\Delta_{s}^{n} \mu\right) \leqq k
$$

for some relatively compact open subset $\Omega$ of $G$, some positive integer $n$, some number $k$, and some relatively compact measurable subset $W$ of $G$ satisfying $m(W)>0$.

We aim to show that as a result

$$
\left\{\int_{G} g(s) \cdot \Delta_{s}^{n} \mu \cdot d s\right\} \mid \Omega \in L^{p}(\Omega)
$$

and therefore, by (2.1.3), that

$$
\left\{\sum_{r=0}^{n}(-1)^{n-r}{ }_{n} C_{r} g_{*}^{(r)} \mu\right\} \mid \Omega \in L^{p}(\Omega),
$$

in each case for any $g \in L_{c}^{\mathbf{1}}(G)$ which vanishes a.e. outside $W$.

To this end, we observe first that the substance of $\S 2.3$ shows that the restrictions $\Delta_{s}^{n} \mu \mid \Omega$ form a bounded subset of $L^{p}(\Omega)$ when $s$ ranges over $W$. Let us verify next that the $L^{p}(\Omega)$-valued function $F$ defined on $W$ by $F(s)=\Delta_{s}^{n} \mu \mid \Omega$ is scalarwise measurable. Granted this, since $p>1$ and therefore $L^{p}(\Omega)$ may be viewed as the dual of $L^{p^{\prime}}(\Omega),(2.4 .2)$ will follow from $[4], 8.16 .1$ and 8.16.3.

As to scalarwise measurability of $F$, observe first that for each 
$\phi \in C_{c}(\Omega)$, the scalar-valued function $s \rightarrow\langle\phi, F(s)\rangle$ is continuous. Given $\psi \in L^{p^{\prime}}(\Omega)$, choose a sequence $\left(\phi_{i}\right)$ from $C_{c}(\Omega)$ (or $C_{c}^{\infty}(\Omega)$, if $G$ is a Lie group) converging in $L^{p^{\prime}}(\Omega)$ to $\psi$; this is possible since $p^{\prime}<\infty$. Then, thanks to the boundedness of $F(W)$ in $L^{p}(\Omega)$, we obtain

$$
\left|\langle\psi, F(s)\rangle-\left\langle\phi_{i}, F(s)\right\rangle\right| \leqq k \cdot\left\|\psi-\phi_{i}\right\|_{p^{\prime}}
$$

uniformly for $s \in W$. It follows that $F$ is scalarwise continuous (a fortiori, scalarwise measurable) on $W$.

2.5 The case $p=1$. The proof of Theorem 5 will demand an analogue of $\S 2.4$ for the case $p=1$, in which it is assumed that

and that

$$
F(s) \equiv \Delta_{s}^{n} \mu \mid \Omega \in L^{1}(\Omega) \quad(s \in W),
$$

$$
N_{1, \Omega}\left(\Delta_{s}^{n} \mu\right) \equiv\|F(s)\|_{L^{1}(\Omega)} \leqq k
$$

and from which it will be necessary to infer that

$$
\left\{\int_{G} g(s) \Delta_{s}^{n} \mu \cdot d s\right\} \mid \Omega=\int_{G} g(s) F(s) d s \in L^{1}(\Omega)
$$

and so, by (2.1.2), that

$$
\left\{\sum_{r=0}^{n}(-1)^{n-r} C_{r} g_{*}^{(r)} \mu\right\} \mid \Omega \in L^{1}(\Omega)
$$

for any $g \in L_{\mathfrak{c}}^{1}(G)$ which vanishes a.e. outside $W$.

Some vital steps in the argument appearing in $\S \mathbf{2 . 4}$ being no longer valid, because in general $L^{1}(\Omega)$ is not a dual space, we are forced to adopt a different approach.

As a beginning, we can show easily from (2.5.1) that

$$
h \equiv \int_{G} g(s) F(s) d s
$$

exists as a bounded measure on $\Omega$ : for this another appeal to 8.16.1 and 8.16 .3 of [4] suffices, regarding the space of bounded measures on $\Omega$ as the dual of $C_{0}(\Omega)$, the space of continuous functions on $\Omega$ which tend to zero at infinity equipped with the supremum norm. The residual problem is to show that, in fact, $h \in L^{1}(\Omega)$. For this we shall again make use of $\S 5$ of [6].

Choose and fix any sequence $\left(\varepsilon_{r}\right)_{r=1}^{\infty}$ of positive numbers tending to zero. For each $s \in W$ and each $r$ we have (since $F(s) \in L^{1}(\Omega)$ ) the existence of a finite infimum $c_{r}(s)$ of numbers $c \geqq 0$ for which

$$
|\langle\phi, F(s)\rangle| \leqq \varepsilon_{r}\|\phi\|_{\infty}+c\|\phi\|_{1}
$$

for all $\phi \in C_{c}(\Omega)$. For $c_{r}(s)$, we have the formula

$$
c_{r}(s)=\operatorname{Sup}\left\{|\langle\phi, F(s)\rangle|-\varepsilon_{r}\|\phi\|_{\infty}\right\},
$$


wherein $\phi$ ranges over all elements of $C_{e}(\Omega)$ satisfying $\|\phi\|_{1}=1$. This formula shows that each function $c_{r}$ is lower semicontinuous and therefore measurable.

Since $c_{r}$ is finite-valued on $W$, given any $\delta>0$, there exists a measurable set $W_{r, \delta} \subset W$ such that $m\left(W_{r, \delta}\right) \geqq m(W)-2^{-r} \delta$ and on which $c_{r}$ is bounded above, say $c_{r}(s) \leqq q_{r, \delta}$ for $s \in W_{r, \delta}$. Putting

$$
W_{\delta}=\bigcap_{r=1}^{\infty} W_{r, \delta},
$$

we shall have $m\left(W_{\delta}\right) \geqq m(W)-\delta$ and $c_{r}(s) \leqq q_{r, \delta}$ for all $r$ and all $s \in W_{\delta}$.

Define $g_{\delta}$ to be equal to $g$ on $W_{\delta}$ and to zero elsewhere, and write

$$
h_{\delta}=\int_{G} g_{\delta}(s) F(s) d s .
$$

Then for all $r$ and all $\phi \in C_{c}(\Omega)$ we shall have

$$
\begin{aligned}
\left|\left\langle\phi, h_{\delta}\right\rangle\right| & \leqq \int_{W_{\delta}}\left|g_{\delta}(s) \|\langle\phi, F(s)\rangle\right| d s \\
& \left.\leqq \int_{W_{\delta}}\left|g_{\delta}(s)\right| \cdot{ }_{\left\{\varepsilon_{r}\right.}\|\phi\|_{\infty}+q_{r, \delta}\|\phi\|_{1}\right\} d s \\
& \leqq \varepsilon_{r}\left\|g_{\delta}\right\|_{1}\|\phi\|_{\infty}+q_{r, \delta}\left\|g_{\delta}\right\|_{1}\|\phi\|_{1}
\end{aligned}
$$

Therefore, by the converse portion of $\S 5$ of $[6], h_{\delta} \in L^{1}(\Omega)$.

Now let $\delta$ range through a sequence of values $\delta_{i} \rightarrow 0$ and write

$$
g_{i}=g_{\delta_{i}}, \quad h_{i}=h_{\delta_{i}}, \quad E_{i}=W_{\delta_{i}} .
$$

From (2.5.1), it follows easily that

$$
\begin{aligned}
\| h_{i}-\left.h_{j}\right|_{L^{1}(\Omega)} & \leqq k \cdot \int_{G}\left|g_{i}-g_{j}\right| d s \\
& \leqq k \cdot \int_{E_{i} \sim E_{j}}|g| d s,
\end{aligned}
$$

where $E_{i} \sim E_{j}$ denotes the symmetric difference of $E_{i}$ and $E_{j}$. Since $m\left(E_{i} \sim E_{j}\right) \rightarrow 0$ as $i, j \rightarrow \infty$, it follows that the sequence $\left(h_{i}\right)$ is convergent in $L^{1}(\Omega)$. Let the limit be $h^{*}$. Then for $\phi \in C_{c}(\Omega)$ it will be the case that

$$
\begin{aligned}
\left\langle\phi, h^{*}\right\rangle & =\lim _{i}\left\langle\phi, h_{i}\right\rangle=\lim _{i} \int_{G} g_{i}(s)\langle\phi, F(s)\rangle d s \\
& =\int_{G} g(s)\langle\phi, F(s)\rangle d s,
\end{aligned}
$$

the last equality following from (2.5.1) and Lebesgue's convergence theorem. It appears thence that $h^{*}=h$, and therefore $h \in L^{1}(\Omega)$. Our goal has thus been reached. 
ReMarks (i) It is immediately apparent that, if $G$ is a Lie group and $\mu \in \mathscr{D}^{\prime}(G)$, one may in the preceding argument replace everywhere $C_{c}(\Omega)$ by $C_{c}^{\infty}(\Omega)$.

(ii) If $G$ is first countable one may proceed otherwise. The final paragraph of $\S 2.4$ will serve to show that $F$ is scalarwise measurable, i.e. that $s \rightarrow\langle\psi, F(s)\rangle$ is measurable for each $\psi \in L^{\infty}(\Omega)$ : this is so because, thanks to our hypothesis on $G$, we can still choose a sequence $\left(\phi_{i}\right)$ from $C_{c}(\Omega)$ (or $C_{c}^{\infty}(\Omega)$, if $G$ is a Lie group) which converges weakly in $L^{\infty}(\Omega)$ to $\psi$. Besides this, first countability of $G$ and relative compactness of $\Omega$ ensure that $L^{1}(\Omega)$ is separable. That $h \in L^{1}(\Omega)$ thus follows from $(2.5 .1)$ on the basis of 8.14.14 and 8.15.2 of [4].

It could also be shown directly that (if $G$ is first countable) the function $F$ is measurable from $W$ into $L^{1}(\Omega)$ (and not merely scalarwise measurable).

\section{The basic lemma}

Once again we shall need to separate the cases $1<p \leqq \infty$ and $p=1$.

3.1 Suppose that $1<p \leqq \infty$ and that $n^{*}$ is a positive integer or $\infty$. Let $\mu \in M(G)$ and define

$$
S=\left\{s \in G: \Delta_{s}^{n} \mu \in L_{\mathrm{loc}}^{p}(G) \text { for some integer } n=n(s) \leqq n^{*}\right\} .
$$

Let $\Omega$ be a relatively compact open subset of $G$.

(a) If $m_{*}(S)>0$ then there exist positive integers $n \leqq n^{*}$ and $k$ so that

$$
N_{p, \Omega}\left(\Delta_{s}^{n} \mu\right) \leqq k
$$

for all $s$ in some compact subset $W$ of $G$ satisfying $m(W)>0$. If, furthermore, $n^{*}=1$, the set $W$ may be assumed to be a neighbourhood of $e$ in $G$.

(b) If $S$ is nonmeagre then there exist positive integers $n \leqq n^{*}$ and $k$ such that (3.1.1) holds for all $s$ in some nonvoid relatively compact open subset $W$ of $G$.

(c) If $G$ is a Lie group, the conclusions (i) and (ii) remain valid if we assume merely that $\mu \in \mathscr{D}^{\prime}(G)$.

Proof. The substance of $\S 2.3$ shows that in all cases we have

$$
S \subset \bigcup\left\{S_{n, k}: n, k=1,2, \cdots, n \leqq n^{*}\right\},
$$

where

$$
S_{n, k}=\left\{s \in G: N_{p, Q}\left(\Delta_{8}^{n} \mu\right) \leqq k\right\} .
$$

Moreover, the definition (2.1.1) of $N_{p, g}$ shows that each set $S_{n, k}$ is closed in $G$; in this connection observe that 


$$
\left\langle\phi, \Delta_{s}^{n} \mu\right\rangle=\left\langle\Delta\left(s^{-1}\right)^{n} \phi, \mu\right\rangle
$$

and that $\Delta_{x}^{n}$ depends continuously on $x \in G$ when regarded as an endomorphism of $C_{c}(G)$ (or of $C_{c}^{\infty}(G)$ if $G$ is a Lie group).

(a) If $m_{*}(S)>0$ it follows from (3.1.2) that $m_{*}\left(S_{n, k}\right)>0$ for some pair $(n, k)$ with $n \leqq n^{*}$. It thus suffices to take for $W$ a suitably large compact subset of $S_{n, k}$.

Consider now the case in which $n^{*}=1$, so that $n$ is forcibly also equal to 1 . The relations

$$
\begin{aligned}
\Delta\left(s^{-1}\right) & =-\tau\left(s^{-1}\right) \Delta(s) \\
\Delta_{s t} & =\tau_{s} \Delta_{t}+\Delta_{s}
\end{aligned}
$$

combine with left translation-invariance of $L_{\mathrm{loc}}^{p}(G)$ to show that $S$ is a subgroup of $G$. Since $m_{*}(S)>0$, Steinhaus' theorem (see, for example, Exercise 4.21 of [4]) entails that $S$ is a neighbourhood of $e$ in $G$.

Choose a symmetric compact neighbourhood $V$ of $e$ in $G$ such that $V \subset S$, and let $U$ be a relatively compact open subset of $G$ containing $\Omega \cdot V^{2}$. According to the substance of $\S 2.3$ we have

$$
\begin{aligned}
V & \subset\left\{s \in V: N_{p, U}\left(\Delta_{s} \mu\right)>\infty\right\} \\
& =\bigcup\left\{s \in V: N_{p, U}\left(\Delta_{s} \mu\right) \leqq k: k=1,2, \cdots\right\} \\
& =\bigcup\left\{S_{k}: k=1,2, \cdots\right\},
\end{aligned}
$$

say. The set $S_{k}$ is closed. Since $V$ is a neighbourhood of $e$, there exists $k$ such that $m\left(S_{k}\right)>0$. Therefore, by Steinhaus' theorem once more, $W=S_{k} \cdot S_{k}^{-1}$ is a neighbourhood of $e$ in $G$.

Let $s \in W$, so that $s=x y^{-1}$ for some $x, y \in S_{k}$. Using (3.1.4), we have

$$
\begin{aligned}
\Delta_{s} \mu=\Delta\left(x y^{-1}\right) \mu & =\tau_{x} \Delta\left(y^{-1}\right) \mu+\Delta_{x} \mu \\
& =-\tau_{x} \tau\left(y^{-1}\right) \Delta_{y} \mu+\Delta_{x} \mu \\
& =-\tau\left(x y^{-1}\right) \Delta_{y} \mu+\Delta_{x} \mu .
\end{aligned}
$$

Hence

$$
\begin{aligned}
N_{p, Q}\left(\Delta_{s} \mu\right) & \leqq N_{p, Q}\left(\tau\left(x y^{-1}\right) \Delta_{x} \mu\right)+N_{p, Q}\left(\Delta_{x} \mu\right) \\
& =N_{p, \tau_{s},}\left(\Delta_{x} \mu\right)+N_{p, Q}\left(\Delta_{x} \mu\right) .
\end{aligned}
$$

Now $\tau_{s} \Omega \subset U$ since $s=x y^{-1} \in V^{2}$; and, of course, $\Omega \subset U$. Therefore

$$
\begin{aligned}
N_{p, \Omega}\left(\Delta_{s} \mu\right) & \leqq N_{p, v}\left(\Delta_{x} \mu\right)+N_{p, U}\left(\Delta_{x} \mu\right) \\
& \leqq k+k \\
& =2 k,
\end{aligned}
$$

and this holds for any $s \in W$. 
(b) If $S$ is nonmeagre, (3.1.2) shows that at least one of the closed sets $S_{n, k}$ has interior points, and we take for $W$ any nonvoid relatively compact open subset of $G$ contained in that $S_{n, k}$.

(c) Only verbal changes are required in the preceding arguments to cover the case in which $G$ is a Lie group and $\mu$ is an arbitrary distribution on $G$, the remaining hypotheses being as before.

3.2 The case $p=1$. Let $n^{*}, S, \mu$ and $\Omega$ be as in $\S 3.1$, except that $p$ is taken equal to 1 . The conclusions are as follows:

(a) if $m_{*}(S)>0$ then there exist positive integers $n \leqq n^{*}$ and $k$ such that

$$
\Delta_{s}^{n} \mu \mid \Omega \in L^{1}(\Omega) \quad \text { and } \quad N_{1, \Omega}\left(\Delta_{s}^{n} \mu\right) \leqq k
$$

for all $s$ in some compact subset $W$ of $G$ satisfying $m(W)>0$. If, furthermore, $n^{*}=1$, the set $W$ may be assumed to be a neighbourhood of $e$ in $G$.

(b) If $n^{*}=1$ and $S$ contains a nonmeagre $F_{\sigma}$-set $S^{\#}$ then there exists a positive integer $k$ such that (3.2.1) holds for $n=1$ and all $s$ in some nonvoid relatively compact open subset $W$ of $G$.

(c) If $G$ is a Lie group, the conclusions (a) and (b) remain valid if we assume merely that $\mu \in \mathscr{D}^{\prime}(G)$.

Proof. (a) We write

$$
S=\bigcup\left\{S_{n, k}: n, k=1,2, \cdots, n \leqq n^{*}\right\},
$$

where

$$
S_{n, k}=\left\{s \in G: \Delta_{s}^{n} \mu \mid \Omega \in L^{1}(\Omega), N_{1, \Omega}\left(\Delta_{s}^{n} \mu\right) \leqq k\right\} .
$$

This time, however, we cannot affirm that $S_{n, k}$ is a closed subset of $G$. Nevertheless, on the basis of $\S 5$ of [6], if we choose and fix a sequence $\left(\varepsilon_{r}\right)_{r=1}^{\infty}$ of positive numbers tending to zero, then we may write

$$
S_{n, k}=\bigcap_{r=1}^{\infty} \bigcup_{q=1}^{\infty} S_{n, k, r, \alpha},
$$

where $S_{n, k, r, q}$ denotes the set of $s \in G$ satisfying

and

$$
N_{1, \Omega}\left(\Delta_{s}^{n} \mu\right) \leqq k
$$

$$
\left|\left\langle\phi, \Delta_{s}^{n} \mu\right\rangle\right| \leqq \varepsilon_{r}\|\phi\|_{\infty}+q\|\phi\|_{1}
$$

for all $\phi \in C_{c}(\Omega)$. (If $G$ is a Lie group and $\mu \in \mathscr{D}^{\prime}(G)$ we restrict $\phi$ to $C_{c}^{\infty}(\Omega)$.) Since it is easy to verify that $S_{n, k, r, q}$ is a closed subset of $G$, the measurability of $S_{n, k}$ is plain.

This being so, the hypothesis $m_{*}(S)>0$ and the relation (3.2.2) combine to show that $m_{*}\left(S_{n, k}\right)>0$ for some pair $(n, k)$; and so, as in $\S 3.1$, we may take for $W$ any sufficiently large compact subset of $S_{n, k}$. 
The case in which $n^{*}=1$ proceeds much as in $\S 3.1$ : in the definition of $S_{k}$ we add the clause " $\Delta_{s} \mu \mid U \in L^{1}(U)$ ", and it is necessary to repeat the argument just given to show that $S_{k}$, although possibly nonclosed, is at any rate measurable.

(b) Write $S^{\#}$ as the union of an increasing sequence $\left(S_{k}^{\not \#}\right)_{k=1}^{\infty}$ of closed subsets of $G$. Then one has

$$
S^{\not \#} \subset \bigcup\left\{S_{k}: k=1,2, \cdots\right\}
$$

where

$$
S_{k}=\left\{s \in S_{k}^{\#}: \Delta_{s} \mu \mid \Omega \in L^{1}(\Omega), N_{1, \Omega}\left(\Delta_{s} \mu\right) \leqq k\right\} .
$$

Since $S_{k}^{\#}$ is closed and contained in $S, S_{k}$ is easily seen to be closed. The relation (3.2.4) then entails that, for some $k, S_{k}$ has a nonvoid interior and we may take for $W$ any nonvoid relatively compact open subset of $S_{k}$.

(c) This requires no special comment: the preceding arguments are applied with $C_{c}(\Omega)$ everywhere replaced by $C_{c}^{\infty}(\Omega)$.

\section{Proofs of theorems 1-5}

In all cases we suppose given any relatively compact open subset $\Omega$ of $G$ and aim to deduce in each case that $\mu \mid \Omega \in L^{p}(\Omega)$. Once this is done, the free choise of $\Omega$ shows that $\mu \in L_{\mathrm{loc}}^{p}(G)$.

4.1 Proof of Theorem 1. By the case $n^{*}=1$ of $3.1(\mathrm{a})$, or by $3.1(\mathrm{~b})$, there exists a number $k$ and a relatively compact open subset $W$ of $G$ such that $m(W)>0$ and

$$
N_{p, \Omega}\left(\Delta_{s} \mu\right) \leqq k
$$

Now apply $\S 2.4$ with $n=1$ : the relation $(2.4 .3)$ yields

that is,

$$
\left\{-g_{*}^{(\mathbf{0})} \mu+g_{*}^{(\mathbf{1})} \mu\right\} \mid \Omega \in L^{p}(\Omega),
$$

$$
\left\{-\int_{G} g(s) d s \cdot \mu+g * \mu\right\} \mid \Omega \in L^{p}(\Omega),
$$

for any $g \in L_{c}^{1}(G)$ which vanishes a.e. outside $W$. In this we take $g \in L_{c}^{p}(G)$ vanishing a.e. outside $W$ and such that $\int_{G} g(s) d s=1$. By $\S 2.2(\mathrm{a})$ and (4.1.2), it follows that $\mu \mid \Omega \in L^{p}(\Omega)$, which relation is our aim.

4.2 Proof of Theorem 2. By $\S 3.1$ (a) or $\S 3.1(\mathrm{~b})$ we see that for some $k$, some positive integer $n$, and some nonnull compact set $W$, we have

$$
N_{p, \Omega}\left(\Delta_{s}^{n} \mu\right) \leqq k \quad(s \in W) .
$$

By 2.4, therefore, 


$$
\left\{\sum_{r=0}^{n}(-1)^{n-r}{ }_{n} C_{r} g_{*}^{(r)} \mu\right\} \mid \Omega \in L^{p}(\Omega)
$$

for any $g \in L_{c}^{\mathbf{1}}(G)$ vanishing a.e. outside $W$. To this relation we now apply $\S 2.2(\mathrm{~b})$, choosing $g \in L_{c}^{p}(G)$ vanishing outside $W$ and such that $\int_{G} g(s) d s=1$. It follows that $\mu \mid \Omega \in L^{p}(\Omega)$ once again.

4.3 Proofs of Theorems 3 And 4. We here rely on $\$ 3.1(c)$. The set $W$ may now be taken to be nonvoid, relatively compact, and open in $G$. The only modification in the arguments appearing under $\S 4.1$ and $\S \mathbf{4 . 2}$ is in the choice of $g$, which is now chosen from $C_{c}^{\infty}(W)$, and in the use of $\S 2.2(\mathrm{c})$ in place of $\S 2.2(\mathrm{a})$ or $\S 2.2(\mathrm{~b})$.

4.4 Proof of Theorem 5. This follows exactly the same type of recipe as was used in $\S \S 4.1-4.3$, with the sole difference that $\S 3.2$ is used in place of $\S 3.1$ and $\S 2.5$ in place of $\S 2.4$.

Consider, for example, the analogue for $p=1$ of Theorem 1 with hypothesis (i). Then $\S 3.2(\mathrm{a})$ or $\S 3.2(\mathrm{~b})$ shows that, for some number $k$ and some compact set $W$ satisfying $m(W)>0$, we have

$$
\Delta_{s} \mu \mid \Omega \in L^{1}(\Omega), N_{1, \Omega}\left(\Delta_{s} \mu\right) \leqq k \quad(s \in w) .
$$

An appeal to $\S 2.5$ shows that (4.1.2) holds with $p=1$, from which point the proof proceeds as in $\S 4.1$.

Similarly for the proof of the analogue for $p=1$ of Theorem 2 with the hypothesis (i).

For the analogue for $p=1$ of Theorem 1 with the hypothesis (ii'), we rely on $\S 3.2(b)$.

The remaining cases call for no comment beyond what has been said under $\S 4.3$ in relation to the case in which $1<p \leqq \infty$.

\section{Some negative results}

5.1 Throughout this section we shall specialise $G$ by assuming it to be a first countable compact Abelian group containing a nonvoid Kronecker set $S$ (see [7], p. 97); $G$ is thus necessarily infinite. It is our aim to show that there exist many true pseudomeasures $\mu$ on $G$ such that $\Delta_{s} \mu$ is a wellbehaved function for each $s \in S$; and that there exist functions $f \notin L^{\infty}(G)$ such that $\Delta_{s} f$ is very well-behaved for each $s \in S$. Concerning pseudomeasures see the Appendices attached to [11], $\S 2.2$ of [8], and also [9]. The constructions depend upon a simple lemma.

5.2 Suppose that $G$ and $S$ are as in 5.1. Then there exist a sequence $\left(\chi_{n}\right)_{n=1}^{\infty}$ of distinct (continuous) characters of $G$ such that $\lim _{n \rightarrow \infty} \chi_{n}=1$ uniformly on $S$. 
Proof. Denote by $\Phi$ the set of continuous complex-valued functions $w$ on $S$ satisfying $|w(s)|=1$ for all $s \in S$. For any $\varepsilon>0$ the set

$$
\Phi_{\varepsilon}=\{W \in \Phi:|w(s)-1| \leqq \varepsilon(s \in S)\}
$$

is evidently uncountable since it contains the constant functions $1-2 c+2 i \sqrt{c(1-c)}$ corresponding to values of the number $c$ satisfying $0 \leqq c \leqq \operatorname{Min}\left(1, \frac{1}{4} \varepsilon^{2}\right)$. On the other hand, since $G$ is first countable, its character group is countable. So, for any $\varepsilon>0$, there exists a function $w \in \Phi_{\varepsilon}$ which coincides on $S$ with no continuous character of $G$.

In particular we can choose a sequence $\left(w_{n}\right)_{n=1}^{\infty}$ from $\Phi$ such that

$$
\left|w_{n}(s)-1\right| \leqq 1 / n
$$

and $w_{n}$ agrees on $S$ with no continuous character of $G$.

We now define the $\chi_{n}(n=1,2, \cdots)$ inductively in the following way. Since $S$ is a Kronecker set, any continuous function on $S$ with absolute value everywhere equal to 1 is the uniform limit on $S$ of continuous characters of $G$. Accordingly we begin by choosing a continuous character $\chi_{1}$ so that

$$
\left|w_{1}(s)-\chi_{1}(s)\right| \leqq 1
$$

Assuming that continuous characters $\chi_{1}, \chi_{2}, \cdots, \chi_{n}$ have been chosen so that

$$
\left|w_{i}(s)-\chi_{i}(s)\right| \leqq 1 / i
$$

for $i \leqq i \leqq n$, and $\chi_{i} \neq \chi_{j}$ whenever $1 \leqq i, j \leqq n$ and $i \neq j$, define

$$
\delta=\operatorname{Inf}_{1 \leqq i \leqq n} \operatorname{Sup}_{s \in S}\left|\chi_{i}(s)-w_{n+1}(s)\right| .
$$

Then $\delta>0$ by choice of the $w_{m}$, and so a continuous character $\chi_{n+1}$ can be chosen so that

$$
\left|w_{n+1}(s)-\chi_{n+1}(s)\right| \leqq \operatorname{Min}\left\{(n+1)^{-1}, \frac{1}{2} \delta\right\} .
$$

It is then easy to see that $\chi_{n+1}$ is different from $\chi_{i}$ for $1 \leqq i \leqq n$, and that the sequence $\left(\chi_{n}\right)_{n=1}^{\infty}$ has all the desired properties as a consequence of (5.2.1) and (5.2.2).

5.3 Let $G$ and $S$ be as in 5.1, and denote by $X$ the character group of $G$. We will show that an infinite sequence $\left(\chi_{n}\right)_{n=1}^{\infty}$ of dictinct characters of $G$ may be chosen from $X$ in such a way that the following is true. Given any sequence $\left(\varepsilon_{n}\right)_{n=1}^{\infty}$ of positive numbers, there exists a true pseudomeasure $\mu$ on $G$ such that, for each $s \in S, \Delta_{s} \mu$ has an absolutely convergent Fourier series and has a Fourier transform $\left(\Delta_{s} \mu\right)^{\wedge}$ satisfying

$$
\begin{aligned}
\left(\Delta_{s} \mu\right)^{\wedge}(\chi) & =0 \text { if } \chi \in X \text { is distinct from all } \chi_{n}, \\
\left(\Delta_{s} \mu\right)^{\wedge}\left(\chi_{n}\right) & =O\left(\varepsilon_{n}\right) \text { as } n \rightarrow \infty .
\end{aligned}
$$


One can even choose $\mu$ so that, in addition, its singular support is equal to $G$ (see $[9]$ ).

Proof. The desired sequence $\left(\chi_{n}\right)$ is obtained by thinning out the sequence constructed in $\S 5.2$ in such a way that $\left\{\chi_{n}: n=1,2, \cdots\right\}$ is a Sidon subset of $X$ (see [7], p. 126), and such that, if

then

$$
\alpha_{n}=\operatorname{Sup}_{s \in S}\left|\chi_{n}(s)-1\right|,
$$

$$
\sum_{n=1}^{\infty} \alpha_{n}<\infty, \alpha_{n} \leqq \varepsilon_{n} \quad(n=1,2, \cdots) .
$$

(a) Take any sequence $\left(c_{n}\right)_{n=1}^{\infty}$ of complex numbers such that

$$
\operatorname{Sup}_{n}\left|c_{n}\right|<\infty, \quad \sum_{n=1}^{\infty}\left|c_{n}\right|^{2}=\infty
$$

and consider the pseudomeasure

$$
\mu=\sum_{n=1}^{\infty} c_{n} \chi_{n} .
$$

Then $\mu$ is a true pseudomeasure on $G$ : if not, in fact, an easy extension of 5.7.7 of [7] would entail that

$$
\sum_{n=1}^{\infty}\left|c_{n}\right|^{2}<\infty
$$

in conflict with (5.3.3). On the other hand,

$$
\Delta_{s} \mu=\sum_{n=1}^{\infty} c_{n}\left(\overline{\chi_{n}(s)}-1\right) \chi_{n}
$$

so that (5.3.1) is evidently satisfied for each $s \in S$ by virtue of (5.3.2), which also ensures that $\Delta_{s} \mu$ has an absolutely convergent Fourier series.

(b) We have still to show that a bounded sequence $\left(c_{n}\right)$ can be chosen so that the pseudomeasure defined by (5.3.4) has its singular support equal to $G$. But suppose this were not the case: let us proceed to derive an absurdity.

A category argument similar to those used in [9] leads from our hypothesis (that $\operatorname{sing} \operatorname{supp} \mu$ is distinct from $G$ for all bounded sequences $\left.\left(c_{n}\right)\right)$ to the conclusion that there exists a fixed nonvoid open subset $U$ of $G$ such that every $\mu$ of the type (5.3.4), with $\left(c_{n}\right)$ a bounded sequence, coincides on $U$ with a measure. By using a partition of unity, it is easily seen that $U$ may be taken to be the whole of $G$; in other words, for any bounded sequence $\left(c_{n}\right)$, the right hand side of (5.3.4) is a Fourier-Stieltjes series. But then ([10], Theorem (1.1)) we may infer that 


$$
\sum_{n=1}^{\infty}\left|c_{n}\right|^{2}<\infty
$$

This conclusion is, it appears, a consequence of the mere boundedness of $\left(c_{n}\right)$, whence the absurdity.

5.4 Next we show that, retaining the notations of $\S 5.3$, there exist functions $f$ such that

$$
f \in L^{p}(G) \quad(1 \leqq p<\infty), f \notin L^{\infty}(G)
$$

and yet, for each $s \in S, \Delta_{s} t$ has an absolutely convergent Fourier series and

$$
\begin{aligned}
& \left(\Delta_{s} f\right)^{\wedge}(\chi)=0 \text { if } \chi \in X \text { is distinct from all } \chi_{n}, \\
& \left(\Delta_{s} f\right)^{\wedge}(\chi)=O\left(\varepsilon_{n}\right) \text { as } n \rightarrow \infty .
\end{aligned}
$$

Proof. We consider sequences $\left(c_{n}\right)$ satisfying

$$
\sum_{n=1}^{\infty}\left|c_{n}\right|^{2}<\infty
$$

and the corresponding functions

$$
f=\sum_{n=1}^{\infty} c_{n} \chi_{n}
$$

Since the $\chi_{n}$ form a Sidon subset of $X,(5.4 .3)$ combines with a known result ([7], 5.7.7) to show that $f \in L^{p}(G)$ for every finite $p$. It is also clear (as in $\S 5.3$ ) that, for each $s \in S, \Delta_{s} f$ has an absolutely convergent Fourier series and satisfies (5.4.2).

It remains to show that $\left(c_{n}\right)$ can be chosen satisfying (5.4.3) and such that $f \notin L^{\infty}(G)$. Now, were such a choice not possible, the uniform boundedness principle (or the closed graph theorem) would entail that

$$
\left\|\sum_{n=1}^{\infty} c_{n} \chi_{n}\right\|_{\infty} \leqq \text { const. }\left\{\sum_{n=1}^{\infty}\left|c_{n}\right|^{2}\right\}^{\frac{1}{2}}
$$

for all such $\left(c_{n}\right)$ and all $h \in L^{1}(G)$. This in turn would show that

$$
\sum_{n=1}^{\infty}\left|\hat{h}\left(\chi_{n}\right)\right|^{2}<\infty
$$

for each $h \in L^{1}(G)$. On the other hand, since the $\chi_{n}$ form a Sidon subset of $X$, the function $h \in L^{1}(G)$ may (see Theorem 5.6 .3 of [7]) be chosen so that the sequence $\left(\hat{h}\left(\chi_{n}\right)\right)$ coincides with any preassigned sequence which converges to zero. This leads to a flat contradiction of (5.4.5) and completes the proof.

5.5 In connection with the results stated in $\S 5.3$ and $\S \mathbf{5 . 4}$, we remark that if $G$ contains arbitrarily small elements of infinite order, then the 
Kronecker set $S$ may be chosen to be perfect and metrisable ([7], pp. $99-100)$; since $G$ is nondiscrete, $S$ is then necessarily uncountable.

On the other hand, if $G$ is the circle group, and if $S$ is any subset of $G$ for which $\S 5.2$ is provable, the positive results established in the present paper show that $S$ must be meagre and of zero measure. In fact it is easy to see that no compact subset $K$ of $S$ can carry any nontrivial measure whose Fourier transform tends to zero at infinity (i.e., $K$ is a set of uniqueness in the wide sense; see $[11]$, pp. 53-57), so that $K$ must have zero generalised capacity relative to any positive convex sequence $\left(\lambda_{k}\right)_{k=1}^{\infty}$ satisfying $\sum_{k=1}^{\infty} \lambda_{k}=\infty$ (see [12], Vol. I, p. 404).

5.6 Assuming still that $G$ is the circle group, similar counterexamples can be constructed for the case in which $S$ is the dense subgroup generated by (the residue classes modulo $2 \pi$ of) any finite number $s_{1}, \cdots, s_{k}$ of real numbers such that at least one of the ratios $s_{j} / 2 \pi(1 \leqq j \leqq k)$ is irrational. For in this case we can choose integers $p_{j n}(1 \leqq j \leqq k, n=1,2, \cdots)$ and $q_{n}(n=1,2, \cdots)$ such that $0<q_{1}<q_{2}<\cdots$ and

$$
\left|s_{j} / 2 \pi-p_{j n} / 2 \pi\right| \leqq q_{n}^{-1-1 / k} \quad(1 \leqq j \leqq k, n=1,2, \cdots) ;
$$

we may suppose furthermore that

$$
\operatorname{Inf}_{n} q_{n+1} / q_{n}>1 .
$$

We then replace the right hand side of $(5.3 .4)$ or $(5.4 .4)$ by

$$
\sum_{n=1}^{\infty} c_{n} \cdot \exp \left(i q_{n} x\right)
$$

If $\left(c_{n}\right)$ satisfies (5.3.3), then (5.6.1) defines a true pseudomeasure $\mu$ on $G$ such that, for each $s \in S, \Delta_{s} \mu$ has an absolutely convergent Fourier series; and $\left(c_{n}\right)$ may be chosen so that sing supp $\mu=G$. Also, $\left(c_{n}\right)$ can be chosen satisfying (5.4.3) and such that the function $t$ defined by (5.6.1) fails to belong to $L^{\infty}(G)$ and yet has the property that, for each $s \in S, \Delta_{s} f$ has an absolutely convergent Fourier series.

By taking $q_{n}=n$ !, it could be arranged that (5.6.1) defines a function $f$ or a pseudomeasure $\mu$ which is itself relatively "wild" and yet which is such that, for any $s$ for which $s / 2 \pi$ is rational, $\Delta_{s} f$ or $\Delta_{s} \mu$ is a trigonometric polynomial.

Yet another variant is obtainable by taking a rapidly increasing sequence $\left(n_{k}\right)_{k=1}^{\infty}$ of positive integers such that $n_{k}$ divides $n_{k+1}$ and

$$
\theta_{k} \equiv n_{k} \sum_{j>k} 1 / n_{j} \rightarrow 0
$$

very rapidly. Consider then a distribution 


$$
f=\sum_{k=1}^{\infty} c_{k} \exp \left(i n_{k} x\right)
$$

If $s$ is the coset modulo $2 \pi Z$ of any real number of the form

$$
s^{\prime}=2 \pi \sum_{k=1}^{\infty} b_{k} / n_{k},
$$

where $\left(b_{k}\right)_{k=1}^{\infty}$ is a bounded sequence of integers, then

and here

$$
\Delta_{s} f=\sum_{k=1}^{\infty} c_{k}\left(\exp \left(i n_{k} s^{\prime}\right)-1\right) \exp \left(i n_{k} x\right),
$$

$$
\exp \left(i n_{k} s^{\prime}\right)-1=\exp \left(2 \pi i n_{k} \sum_{j>k} b_{j} / n_{j}\right)-1=O\left(\theta_{k}\right) .
$$

It is thus clear that $\Delta_{s} f$ can be made a very well-behaved function for each of the uncountably many cosets $s$ defined by the real numbers $s^{\prime}$ of the form (5.6.3). (Incidentally, if the $n_{k}$ be suitably chosen, all but countably many of the numbers $s^{\prime} / 2 \pi$ will be transcendental Liouville numbers.)

5.7 The preceding constructions depend quite crucially on the circumstance that $\mu$ and $f$ possess lacunary Fourier series. For this very reason, however, they fail to procedure examples of functions (or even of measures) $f$ such that, for some finite $p, \Delta_{s} f \in L^{p}(G)$ for infinitely many $s \in G$ and yet $f \notin L^{p}(G)$. Can this phenomenon actually occur?

\section{References}

[1] N. G. de Bruijn, 'Functions whose differences belong to a given class', Nieuw Arch. Wiskunde (2) 23 (1951), $194-218$.

[2] N. G. de Bruijn, 'A difference property for Riemann integrable functions and for some similar classes of functions, Nederl. A kad. Wetensch. Proc. Ser. A 55 (1952), 145- 151.

[3] R. E. Edwards, 'Translates of $L_{\infty}$ functions and of bounded measures', J. Austr. Math. Soc. 4 (1964), $304-409$.

[4] R. E. Edwards, Functional Analysis: Theory and Applications (Holt, Rinehart \& Winston, Inc., New York, 1965).

[5] L. Schwartz, Théorie des Distributions, Tomes I, II (Hermann et Cie, Paris, 1950, 1951).

[6] R. E. Edwards, 'Criteria for Fourier transforms'. J. Austr. Math. Soc. 7 (1967), $239-246$.

[7] W. Rudin, Fourier analysis on groups (Interscience Publishers, New York, 1962).

[8] R. E. Edwards, 'Uniform approximation on noncompact spaces'. Trans. Amer. Math. Soc. 122 (1966), $249-276$.

[9] R. E. Edwards, 'Supports and singular supports of pseudomeasures', J. Austr. Math. Soc. 6 (1966), $65-75$.

[10] R. E. Edwards, 'Changing signs of Fourier coefficients', Pacific J. Math. 15 (1965), $463-475$.

[11] J.-P. Kahane et R. Salem, Ensembles parfaits et séries trigonométriques (Hermann et Cie, Paris, 1963).

[12] N. Bary, A treatise on trigonometric series, Vols. I, II (Pergamon Press, Oxford, 1964 ).

[13] E. Hewitt and K. A. Ross, Abstract Harmonic Analysis, I (Springer-Verlag, BerlinGöttingen-Heidelberg, 1963). 
[14] J. H. B. Kemperman, 'A general functional equation', Trans. A mer. Math. Soc. 86 (1957), 28-56.

[15] F. W. Carroll, 'Functions whose differences belong to $L^{p}(0,1)$ ', Nederl. Akad. Wetensch. Proc. Ser. A 67 (1964), 250-255.

[16] F. W. Carroll, 'A difference property for polynomials and exponential polynomials on abelian locally compact groups', Trans. Amer. Math. Soc. 114 (1965), 147-155.

Department of Mathematics

Institute of Advanced Studies

Australian National University 